PROCEEDINGS OF THE

AMERICAN MATHEMATICAL SOCIETY

Volume 129, Number 6, Pages 1733-1737

S 0002-9939(01)05792-6

Article electronically published on January 17, 2001

\title{
LOCAL DERIVATIONS OF REFLEXIVE ALGEBRAS II
}

\author{
JING WU
}

(Communicated by David R. Larson)

\begin{abstract}
Let $\mathcal{A}$ be a reflexive algebra in Banach space $X$ such that both $0_{+} \neq 0$ and $X_{-} \neq X$ in Lat $\mathcal{A}$. Then every local derivation of $\mathcal{A}$ into itself is a derivation.
\end{abstract}

\section{INTRODUCTION}

When attempting to find sufficient conditions for a linear mapping to be a derivation, an obvious candidate is the concept of a local derivation. Let $\mathcal{B}$ be a Banach algebra. We say that a linear transformation $\phi: \mathcal{B} \rightarrow \mathcal{B}$ is a local derivation if for every $B \in \mathcal{B}$ there is a derivation $\delta_{B}: \mathcal{B} \rightarrow \mathcal{B}$ depending on $B$, such that $\phi(B)=\delta_{B}(B)$. The theory of local derivation originates from Kadison's theorem, which says that each norm-continuous local derivation of a von Neumann algebra $\mathcal{B}$ on Hilbert space into a dual $\mathcal{B}$-bimodule $\mathcal{M}$ is a derivation. In the last few years interest in local derivations of operator algebras in Banach space has also been growing.

In [6] Larson and Sourour proved that every local derivation of $B(X)$ into itself is a derivation, where $B(X)$ denotes the algebra of all bounded linear operators on a complex Banach space $X$.

Han Deguang and Wei Shuyun [2] showed that every norm-continuous local derivation of some nest algebras is a derivation.

In our previous publication 3] we proved that if $\mathcal{A}$ is a reflexive algebra in reflexive Banach space $X$ such that both $0_{+} \neq 0$ and $X_{-} \neq X$ in Lat $\mathcal{A}$, then every norm-continuous local inner derivation of $\mathcal{A}$ into itself is a derivation.

We ought perhaps to mention that the conditions in 3. such as the reflexivity of $X$ and the assumptions that $\delta$ is continuous and inner are very strong. It is the purpose of this paper to drop the assumptions on $X$ and $\delta$ in the result of [3], thus extending the result to a more general setting. More precisely, we shall see that every local derivation of $\mathcal{A}$ into itself is a derivation. Note that our approach is quite different from that of [3] but is simple.

Received by the editors September 18, 1998 and, in revised form, January 6, 1999 and September 20, 1999.

2000 Mathematics Subject Classification. Primary 47L10, 47B47.

Key words and phrases. Reflexive algebra, derivation, local derivation.

This project was supported by the NNSF of China.

(C)2001 American Mathematical Society 


\section{Preliminaries AND notations}

In what follows we denote by $X$ a fixed complex Banach space. The usual notation Lat $\mathcal{B}$ will denote the lattice of invariant subspaces for a subset $\mathcal{B} \subseteq B(X)$, and $\operatorname{Alg} \mathcal{L}$ will denote the algebra of bounded linear operators leaving invariant every member of a family $\mathcal{L}$ of subspaces. $\mathcal{B}$ is reflexive if $\mathcal{B}=$ ref $\mathcal{B}$, where ref $\mathcal{B}=\{T \in B(X): T x \in[\mathcal{B} x], x \in X\}$ and [.] denotes the norm closure.

For a lattice $\mathcal{L}$ of subspaces of $X$, if $N \in \mathcal{L}$, we denote $\bigvee\{M \in \mathcal{L}: N \nsubseteq \mathbb{}$ \} by $N_{-}$and $\bigwedge\{M \in \mathcal{L}: M \nsubseteq N\}$ by $N_{+}$.

For a subset $\mathcal{S} \subseteq X, \mathcal{S}^{\perp}=\left\{f \in X^{*}: f(\mathcal{S})=\{0\}\right\}$, where $X^{*}$ is the dual space of $X$. If $x \in X$ and $f \in X^{*}$, the rank one operator $u \mapsto f(u) x$ is denoted by $x \otimes f$.

Let $L(X)$ denote the algebra of all linear transformations from $X$ into itself. If $\mathcal{I}$ is a subset of $B(X)$, we write $\operatorname{ref}_{a}(\mathcal{I})=\{T \in L(X): T x \in \mathcal{I} x, x \in X\}$, where $\mathcal{I} x=\{S x: S \in \mathcal{I}\}$. The set $\mathcal{I}$ is said to be algebraically reflexive if $\mathcal{I}=\operatorname{ref}_{a}(\mathcal{I})$.

The following lemma is taken from [7]; it will get repeated use.

Lemma 2.1. If $\mathcal{L}$ is a subspace lattice, then $x \otimes f \in A l g \mathcal{L}$ if and only if there exists an element $L \in \mathcal{L}$ such that $x \in L$ and $f \in\left(L_{-}\right)^{\perp}$.

\section{LOCAL DERIVATIONS}

Throughout this section, $\mathcal{A}$ will be a reflexive algebra in Banach space $X$ such that both $0_{+} \neq 0$ and $X_{-} \neq X$ in Lat $\mathcal{A}$.

Lemma 3.1. For $A \in \mathcal{A}$,

(1) if $R A=0$ for every rank one operator $R \in \mathcal{A}$ of the form $x \otimes f$ with $x \in 0_{+}$ and $f \in X^{*}$, then $A=0$;

(2) if $A R=0$ for every rank one operator $R \in \mathcal{A}$ of the form $x \otimes f$ with $x \in X$ and $f \in\left(X_{-}\right)^{\perp}$, then $A=0$.

The proof of this lemma is straightforward so we omit it.

The following lemma can be found in $[8]$ but we present the proof for the sake of completeness.

Lemma 3.2. Let $\delta$ be a local derivation from a Banach algebra $\mathcal{B}$ into a $\mathcal{B}$-module $\mathcal{M}$. Then $\delta(P A Q)=\delta(P A) Q+P \delta(A Q)-P \delta(A) Q$ holds for each $A \in \mathcal{B}$ and any idempotents $P$ and $Q$ in $\mathcal{B}$.

Proof. Let $A \in \mathcal{B}$. As $\delta$ is a local derivation, it is easy to see that

$$
P^{\perp} \delta(P A Q) Q^{\perp}=0
$$

for any idempotents $P, Q$ in $\mathcal{B}$, where $P^{\perp}=I-P$ and $Q^{\perp}=I-Q$ are also idempotents. Since

$$
\begin{aligned}
\delta(P A Q) Q^{\perp}-P \delta(A Q) Q^{\perp} \\
\quad=[\delta(P A Q)-P \delta(A Q)] Q^{\perp} \\
\quad=\left[\left(P^{\perp} \delta(P A Q)+P \delta(P A Q)\right)-\left(P \delta\left(P^{\perp} A Q\right)+P \delta(P A Q)\right)\right] Q^{\perp} \\
\quad=P^{\perp} \delta(P A Q) Q^{\perp}-P \delta\left(P^{\perp} A Q\right) Q^{\perp} \\
\quad=0,
\end{aligned}
$$

hence

$$
\delta(P A Q) Q^{\perp}=P \delta(A Q) Q^{\perp}
$$


Since $Q^{\perp}=I-Q$ is also an idempotent, similarly we have

$$
\delta\left(P A Q^{\perp}\right) Q=P \delta\left(A Q^{\perp}\right) Q .
$$

Then we obtain that

$$
\begin{aligned}
\delta(P A Q)-P \delta(A Q) & =(\delta(P A Q)-P \delta(A Q)) Q^{\perp}+(\delta(P A Q)-P \delta(A Q)) Q \\
& =(\delta(P A Q)-P \delta(A Q)) Q \\
& =\left[\left(\delta(P A)-\delta\left(P A Q^{\perp}\right)\right)-\left(P \delta(A)-P \delta\left(A Q^{\perp}\right)\right)\right] Q \\
& =\delta(P A) Q-P \delta(A) Q+\left(P \delta\left(A Q^{\perp}\right) Q-\delta\left(P A Q^{\perp}\right) Q\right) \\
& =\delta(P A) Q-P \delta(A) Q
\end{aligned}
$$

and so $\delta(P A Q)=\delta(P A) Q+P \delta(A Q)-P \delta(A) Q$.

Theorem 3.3. Let $\mathcal{A}$ be a reflexive algebra in Banach space $X$ such that both $0_{+} \neq 0$ and $X_{-} \neq X$ in LatA. Then every local derivation of $\mathcal{A}$ into itself is a derivation.

Proof. Let $\delta: \mathcal{A} \rightarrow \mathcal{A}$ be a local derivation. Then $\delta(I)=0$. By Lemma 3.2 we have that

$$
\delta(P Q)=\delta(P) Q+P \delta(Q)
$$

holds for any rank one projections $P$ and $Q$ in $\mathcal{A}$.

To prove that $\delta(A B)=\delta(A) B+A \delta(B)$ for every $A, B \in \mathcal{A}$, we divide the proof into several steps.

Step 1. For any rank one operator $P, Q \in \mathcal{A}$, where $P=x \otimes f$ with $x \in 0_{+}$and $f \in X^{*}, Q=y \otimes g$ with $y \in X$ and $g \in\left(X_{-}\right)^{\perp}$, we have $\delta(P Q)=\delta(P) Q+P \delta(Q)$.

Case 1. If $f(x) \neq 0$ and $g(y) \neq 0$. Let $P^{\prime}=P / f(x)$ and $Q^{\prime}=Q / g(y)$. Then both $P^{\prime}$ and $Q^{\prime}$ are rank one projections, so we have

$$
\delta\left(P^{\prime} Q^{\prime}\right)=\delta\left(P^{\prime}\right) Q^{\prime}+P^{\prime} \delta\left(Q^{\prime}\right)
$$

By the linearity of $\delta$, we obtain that $\delta(P Q)=\delta(P) Q+P \delta(Q)$.

Case 2. If one of $f(x)$ and $g(y)$ is 0 . Without loss of generality, suppose that $f(x)=0$ and $g(y) \neq 0$. Choose $f^{\prime} \in X^{*}$ such that $\left(f+f^{\prime}\right)(x)=f^{\prime}(x) \neq 0$. Then by Case 1 ,

$$
\begin{aligned}
\delta(P Q) & =\delta\left(x \otimes\left(f+f^{\prime}\right) \cdot y \otimes g\right)-\delta\left(x \otimes f^{\prime} \cdot y \otimes g\right) \\
& =\delta\left(x \otimes\left(f+f^{\prime}\right)\right) y \otimes g+x \otimes\left(f+f^{\prime}\right) \delta(y \otimes g)-\delta\left(x \otimes f^{\prime}\right) y \otimes g-x \otimes f^{\prime} \delta(y \otimes g) \\
& =\delta(x \otimes f) y \otimes g+x \otimes f \delta(y \otimes g) \\
& =\delta(P) Q+P \delta(Q) .
\end{aligned}
$$

Case 3. If both $f(x)$ and $g(y)$ are 0 . Choose $f^{\prime} \in X^{*}$ and $y^{\prime} \in X$ such that $\left(f+f^{\prime}\right)(x)=f^{\prime}(x) \neq 0$ and $g\left(y+y^{\prime}\right)=g\left(y^{\prime}\right) \neq 0$. With the same argument as in Case 2, we also have that $\delta(P Q)=\delta(P) Q+P \delta(Q)$.

Step 2. For each $A \in \mathcal{A}$ and any rank one operator $Q \in \mathcal{A}$, we have

$$
\delta(A Q)=\delta(A) Q+A \delta(Q)
$$

where $Q=y \otimes g$ with $y \in X$ and $g \in\left(X_{-}\right)^{\perp}$.

For any rank one operator $P \in \mathcal{A}$ of the form $x \otimes f$ with $x \in 0_{+}$and $f \in X^{*}$, by using an argument similar to that used in Step 1, we know that Lemma 3.2 holds for each $A \in \mathcal{A}$ and any rank one operators $P, Q \in \mathcal{A}$, where $P=x \otimes f$ with $x \in 0_{+}$ and $f \in X^{*}, Q=y \otimes g$ with $y \in X$ and $g \in\left(X_{-}\right)^{\perp}$. 
On the other hand, by Step 1,

$$
\delta(P A Q)=\delta(P A \cdot Q)=\delta(P A) Q+P A \delta(Q) .
$$

Then we get that

$$
P \delta(A Q)=P \delta(A) Q+P A \delta(Q) ;
$$

by Lemma 3.1, this yields that

$$
\delta(A Q)=\delta(A) Q+A \delta(Q) .
$$

Step 3. For any $A, B \in \mathcal{A}$, we have that $\delta(A B)=\delta(A) B+A \delta(B)$, i.e. $\delta$ is a derivation. 2 ,

For each rank one operator $Q=y \otimes g \in \mathcal{A}$ with $y \in X$ and $g \in\left(X_{-}\right)^{\perp}$, by Step

$$
\delta(A B Q)=\delta(A B) Q+A B \delta(Q) .
$$

On the other hand, also by Step 2,

$$
\delta(A B Q)=\delta(A \cdot B Q)=\delta(A) B Q+A \delta(B Q)=\delta(A) B Q+A \delta(B) Q+A B \delta(Q) .
$$

Thus we have that $\delta(A B) Q=\delta(A) B Q+A \delta(B) Q$, i.e. $(\delta(A B)-\delta(A) B-A \delta(B)) Q=$ 0 .

By Lemma 3.1, we obtain that $\delta(A B)=\delta(A) B+A \delta(B)$, i.e. $\delta$ is a derivation. This completes the proof.

Corollary 3.4. The set of all derivations of $\mathcal{A}$ into itself is algebraically reflexive.

Corollary 3.5. If $\mathcal{N}$ is a nest on $X$ such that both $0_{+} \neq 0$ and $X_{-} \neq X$ in $\mathcal{N}$, then the set of all derivations of nest algebra AlgN $\mathcal{N}$ into itself is algebraically reflexive.

Corollary 3.6. If $\mathcal{L}$ is a commutative subspace lattice on $X$ such that both $0_{+} \neq 0$ and $X_{-} \neq X$ in $\mathcal{L}$, then the set of all derivations of the CSL algebra AlgL into itself is algebraically reflexive.

For the local derivations of standard algebras we have

Corollary 3.7. If $\mathcal{B}$ is a standard operator algebra in $X$, then every local derivation of $\mathcal{B}$ into itself is a derivation.

The following corollary is well known.

Corollary 3.8. Every local derivation of $B(X)$ is a derivation.

\section{ACKNOWLEDGMENT}

The author is grateful to Professors Gong Weibang and Han Deguang for their encouragment. He also expresses his thanks to the referee for many suggestions.

\section{REFERENCES}

[1] D. Hadwin, Algebraically reflexive linear transformations, Linear and Multilinear Algebra 14 (1983), 225-233. MR 85e:47003

[2] Han Deguang and Wei Shuyun, Local derivations of nest algebras, Proc. Amer. Math. Soc. 123(1995), 3095-3100. MR 95m:47077

[3] Jing Wu, Local derivations of reflexive algebeas, Proc. Amer. Math. Soc. 125(1997), 869-873. MR 97e: 47073

[4] R. V. Kadison, Local derivations, J. Algebras 130 (1990), 494-509. MR 91f:46092 
[5] D. R. Larson, Reflexivity, algebraic reflexivity and linear interpolation, Amer. J. Math. 110(1988), 283-299. MR 89d:47096

[6] D. R. Larson and A. R. Sourour, Local derivations and local automorphisms of $B(X)$, Proc. Sym. Pure Math. 51 (1990), 187-194. MR 91k:47106

[7] W. E. Longstaff, Strongly reflexive lattices, J. London Math. Soc. 119(1975), 491-498. MR 52:15036

[8] Xu Benlong and Ma Jipu, A note on local derivation, Adv. Math (China) (in Chinese) 27(1998), 45-46. MR 99g:46096

Department of Mathematics, Yantai Teachers' College, Yantai, Shandong, 264025, People's Republic of China

E-mail address: jingwu@public.ytptt.sd.cn

Current address: Department of Mathematics, Yuquan Campus of Zhejiang University, Hangzhou, Zhejiang 310027, People's Republic of China

E-mail address: jingwu@math.zju.edu.cn 